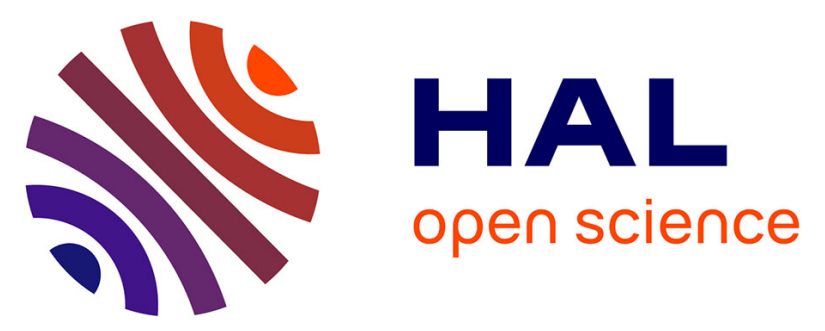

\title{
Frottement des matériaux composites polymères à renfort fibre de carbone: expériences et modélisation
}

Olga Smerdova, Alain Le Bot, Juliette Cayer-Barrioz, Boris Sarbaev

\section{To cite this version:}

Olga Smerdova, Alain Le Bot, Juliette Cayer-Barrioz, Boris Sarbaev. Frottement des matériaux composites polymères à renfort fibre de carbone: expériences et modélisation. 17èmes Journées Nationales sur les Composites (JNC17), Jun 2011, Poitiers-Futuroscope, France. pp.178. hal-00597754

\section{HAL Id: hal-00597754 \\ https://hal.science/hal-00597754}

Submitted on 1 Jun 2011

HAL is a multi-disciplinary open access archive for the deposit and dissemination of scientific research documents, whether they are published or not. The documents may come from teaching and research institutions in France or abroad, or from public or private research centers.
L'archive ouverte pluridisciplinaire HAL, est destinée au dépôt et à la diffusion de documents scientifiques de niveau recherche, publiés ou non, émanant des établissements d'enseignement et de recherche français ou étrangers, des laboratoires publics ou privés. 


\title{
Frottement des matériaux composites polymères à renfort fibre de carbone : expériences et modélisation
}

\section{Friction between carbon fiber reinforced polymers : experiments and modelling}

\author{
Olga Smerdova ${ }^{1,2^{*}}$, Alain le Bot ${ }^{1}$, Juliette Cayer-Barrioz ${ }^{1}$ et Boris Sarbaev ${ }^{2}$ \\ 1 : Laboratoire de Tribologie et Dynamique des Systèmes, CNRS UMR 5513, Ecole Centrale de Lyon, \\ 36 avenue Guy de Collongue, 69134 ECULLY CEDEX, FRANCE \\ 2 : Chaire SM1, Université Technique d'Etat de Moscou Bauman, 5, 2-nd Baumanskaya, 105005, MOSCOU, RUSSIE \\ *e-mail : olga.smerdova@ec-lyon.fr
}

\begin{abstract}
Résumé
Les matériaux composites polymères renforcés avec des fibres de carbone (PRFC) sont connus pour l'excellente combinaison de leurs propriétés mécaniques et thermiques avec leur faible masse. Cependant, leurs propriétés tribologiques restent largement méconnues. Dans le cadre de ce travail, l'étude expérimentale du frottement entre deux PRFC composites sous faible charge normale (jusqu'à 20N) a été réalisée. Deux effets ont été soigneusement étudiés pendant l'expérience : la fraction volumique et l'orientation des fibres. En complément de ce travail expérimental, une modélisation du contact entre deux PRFC a été réalisée. On suppose que l'aire réelle de contact est constituée d'une multitude de microcontacts de trois types : fibre-fibre, fibre-matrice et matrice-matrice. L'étude expérimentale a montré une légère augmentation de coefficient de frottement avec le changement d'orientation de fibre de deux composites de parallèle à perpendiculaire par rapport au sens de glissement, alors que le modèle analytique proposé prédit une indépendance de cet angle. Quant à l'influence de la fraction volumique de fibres, $V_{f}$, les essais soulignent une diminution de coefficient de frottement de moitié avec une augmentation de $V_{f}$ de $0 \%$ à $62 \%$, ce qui correspond à la dépendance qualificative trouvée dans le cadre du modèle.
\end{abstract}

\begin{abstract}
Carbon fiber reinforced polymers (CFRP) are well-known for the excellent combination of mechanical and thermal properties with light weight. However, their tribological properties are still largely uncovered. In this work an experimental study of friction between two CFRP at weak normal load (inferior to $20 \mathrm{~N}$ ) was performed. Two effects were scrutinuously studied during the experiments: fiber volume friction and fiber orientation. In addition to this experimental work, a modelling of a contact between two FRP was realized. It is supposed that the real area of contact consists of a multitude of microcontacts of three types: fibre-fibre, fibre-matrix and matrix-matrix. The experimental work has shown a small rise of friction coefficient with the change of fiber orientation of two composites from parallel to perpendicular relative to the sliding direction. In parallel, the proposed analytical model predicts an independence on this angle. Regarding the influence of the fiber volume fraction, $V_{f}$, the experiments reveal a decrease in friction coefficient of $50 \%$ with a change of $V_{f}$ from $0 \%$ to $62 \%$. This observation corresponds to the qualitative dependence depicted with the model.
\end{abstract}

Mots Clés : polymère à renfort fibre de carbone, frottement sec, aire de contact réelle, loi de mélange Keywords : carbon fiber reinforced polymer, dry friction, composite materials, real contact area, low of mixtures

\section{Introduction}

Les matériaux composites polymères à renfort fibre de carbone sont utilisés aussi bien dans l'industrie des loisirs que dans l'industrie aéronautique et spatiale en raison de la combinaison de ces excellentes propriétés mécaniques et thermiques avec un faible poids. Ces propriétés tribologiques en contact avec des métaux ou un saphir $[1,2]$ sous conditions d'usure ont été également étudiées depuis des décennies. Plusieurs auteurs ont cherché expérimentalement les effets de la charge normale, la vitesse, les conditions environnementales, etc. sur le coefficient de frottement et l'usure des composites renforcés par fibres de carbone à haut module et à haute résistance mécanique [2-5]. Certains ont proposé des modèles numériques d'un contact entre un composite fibreux et une aspérité de corps opposé [6]. Les effets de la fraction volumique et de l'orientation des fibres ont été 
considérés [7-9]. Tous ces travaux décrivent le frottement sous charge normale suffisamment forte pour induire une usure du composite.

Le coefficient de frottement du contact composite fibreux/antagoniste est obtenu à partir de coefficients de frottement de ses composants selon la loi de mélange suivante [10]

$$
\frac{1}{\mu}=\frac{V_{f}}{\mu_{f}}+\frac{V_{m}}{\mu_{m}}
$$

où $V_{f}$ et $V_{m}$ sont les fractions volumiques, $\mu_{f}$ et $\mu_{m}$ sont les coefficients de frottement des matériaux de fibre et de la matrice respectivement.

Une autre loi est proposée par Axen et al. [11] pour le frottement abrasif de matériaux multiphasiques. Si les pressions normales sont égales sur toutes les phases du composite, on peut calculer le coefficient de frottement ainsi :

$$
\mu=A_{m} \mu_{m}+A_{f} \mu_{f}
$$

où $A_{m}$ et $A_{f}$ sont les fractions surfaciques de matrice et de fibres.

Ces deux lois, ainsi que les travaux expérimentaux cités ci-dessus, décrivent le frottement entre un composite et un matériau isotrope.

L'objectif fixé dans ce travail est d'étudier le frottement de deux polymères à renfort fibre de carbone. Nous nous sommes concentrés sur les effets « composite », c'est-à-dire l'orientation et la fraction volumique de fibres. Le coefficient de frottement est défini par la loi de Coulomb, comme le rapport entre la force tangentielle et la force normale. La deuxième partie de ce travail a consisté à établir un modèle analytique permettant de prédire le coefficient de frottement entre deux composites fibreux.

\section{Etude expérimentale}

\subsection{Description des essais}

\section{Le dispositif}

Le travail expérimental s'est déroulé sur le tribomètre RA conçu et développé au LTDS, dont le schéma de principe est représenté à la fig.1 et dont les caractéristiques sont données dans tab.1. Ce tribomètre permet de réaliser un mouvement linéaire alternatif entre deux échantillons plans de relativement grande surface et de mesurer la force de frottement simultanément.

Pour mener l'essai de frottement, deux types d'échantillons ont été fabriqués : des pistes, échantillons rectangulaires longs, fixés dans le tribomètre, et des glisseurs, échantillons ronds, posés en contact avec les pistes et poussés par un bras linéaire. La charge normale est appliquée par l'intermédiaire d'un poids posé sur le glisseur. Le mouvement du bras linéaire est dirigé par un servomoteur sans balais. La vitesse de rotation du moteur est mesurée par un décodeur et est maintenue constante par une boucle de rétroaction avec un variateur électrique. La précision de la vitesse est de $1 \%$. Pendant un essai, la force tangentielle est mesurée continuement par un capteur piézoélectrique fixé dans le bras linéaire. Ensuite le signal est amplifié par un amplificateur de charge avant son acquisition par un PC.

\begin{tabular}{|c|c|c|c|c|c|}
\hline $\begin{array}{c}\text { Type de } \\
\text { contact }\end{array}$ & $\begin{array}{c}\text { Conditions } \\
\text { environnementaux }\end{array}$ & $\begin{array}{c}\text { Type de } \\
\text { motion }\end{array}$ & $\begin{array}{c}\text { Gamme de } \\
\text { vitesse }\end{array}$ & $\begin{array}{c}\text { Distance de } \\
\text { glissement }\end{array}$ & $\begin{array}{c}\text { Charge } \\
\text { normale }\end{array}$ \\
\hline Plan/plan & ambiantes & $\begin{array}{c}\text { Linéaire, } \\
\text { aller-retour }\end{array}$ & $0.1-200 \mathrm{~mm} / \mathrm{s}$ & jusqu'à $500 \mathrm{~mm}$ & jusqu'à $20 \mathrm{~N}$ \\
\hline
\end{tabular}

Tab. 1. Les caractéristiques générales du tribomètre $R A$ 

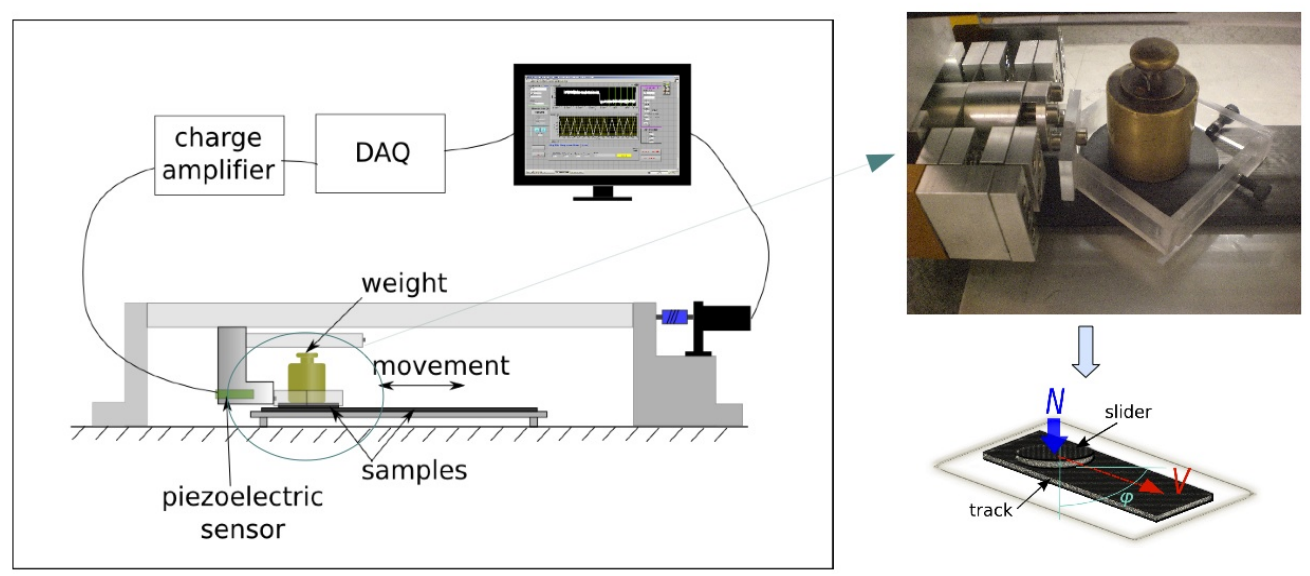

Fig. 1. Tribomètre RA. Schéma du dispositif.

\section{Les matériaux}

Les échantillons ont été fabriqués en quatre options distingués par la fraction volumique de fibres : résine pure, $34 \%, 52 \%$ et $62 \%$ de fibres. Dans tous les cas le matériau était composé par des couches de préimprégné (HexPly $\left.{ }^{\circledR} M 10 / 38 \% / U D 300 / C H S\right)$ de $62 \%$ de fibres $(H e x T o w \circledR A S 4)$ et une résine époxy (HexPly ${ }^{\circledR}$ M10.1) en proportion différente. Ainsi la fraction volumique totale d'un échantillon varie en fonction du nombre de couches et de la quantité de résine. Toutes les couches de préimprégné ont la même orientation de fibre. Cependant l'alignement des couches perpendiculairement à la surface frottante n'est pas contrôlé : ainsi, les échantillons de $34 \%$ et $52 \%$ ont une surface contenant des zones de préimprégné et des zones d'époxy pure (fig. 2). Ceci ne permet pas de considérer la surface comme homogène, mais fournit un ordre de grandeur qualitatif du pourcentage de fibres sur la surface.

Fraction volumique de fibre nominale: $34 \%$
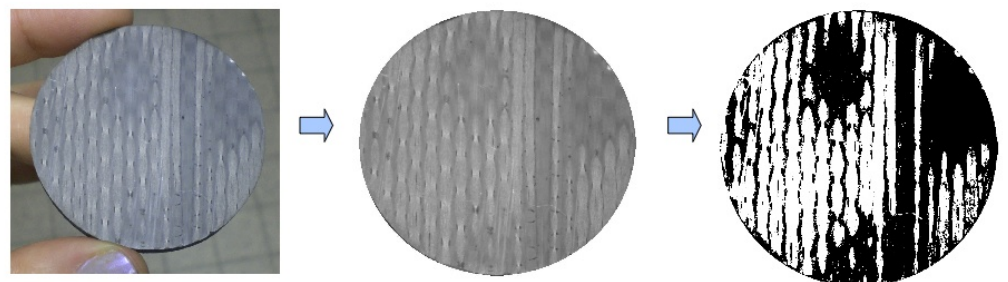

Fraction volumique de fibre nominale: $\mathbf{5 2} \%$
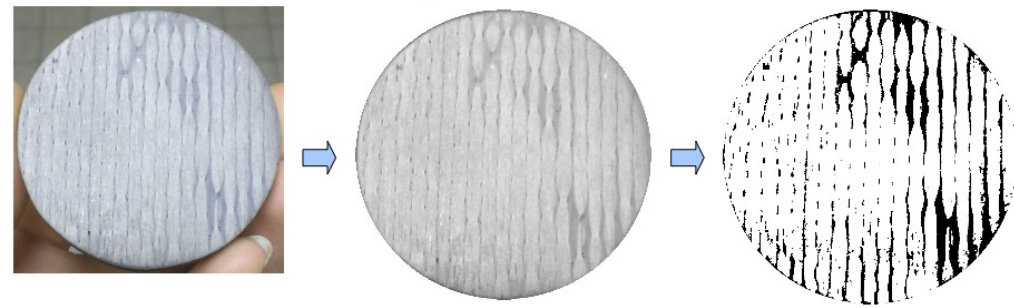

Fig. 2. Glisseurs de $34 \%$ et $52 \%$. Sur image traitée (à droite) : noir-époxy pure, blanc-préimprégné de $62 \%$ de fibres.

\section{Préparation des surfaces}

Les tailles des échantillons sont de $200 \times 50 \times 5 \mathrm{~mm}^{3}$ et $\varnothing 50 \times 5 \mathrm{~mm}^{3}$, respectivement pour une piste et un glisseur. Afin d'améliorer l'état de surface initial, les glisseurs ont été polis avec un papier de polissage $1200 \mathrm{P}$. En raison de leurs grandes dimensions, les pistes n'ont pas pu être polies et leur surface est restée légèrement ondulée. Avant de commencer les essais, les deux surfaces en contact 
sont soigneusement nettoyées avec de l'heptane, de l'acétone et du propanol-2 successivement et enfin avec un flux d'azote.

\section{Les conditions d'un essai}

L'humidité et la température ambiantes ont été contrôlées pendant les essais. Des études préliminaires ont montré que le changement de la vitesse de glissement dans un intervalle de 0.1 à $200 \mathrm{~mm} / \mathrm{s}$ n'a pas d'influence sur le coefficient de frottement entre deux PRFC. Quant à la charge normale, elle a été maintenue constante à $2 \mathrm{~N}$ pour tous les essais comparatifs entre les composites et époxy pure afin de s'affranchir de l'usure importante générée sur les échantillons en epoxy pure sous plus forte charge normale. Pour chaque paire de matériau, l'essai de frottement est répété au moins trois fois.

\subsection{Résultats expérimentaux}

Chaque essai est limité par la distance de glissement de $100 \mathrm{~mm}$ et le nombre de cycles d'allerretour compris entre 20 et 50, dépendant de la répétitivité d'essai. Les moyennes des coefficients de frottement sont déterminées pour tous les essais. Les résultats principaux, les effets de la fraction volumique de fibres et l'orientation de fibres sur le coefficient de frottement, sont présentés aux fig. 3,4 .

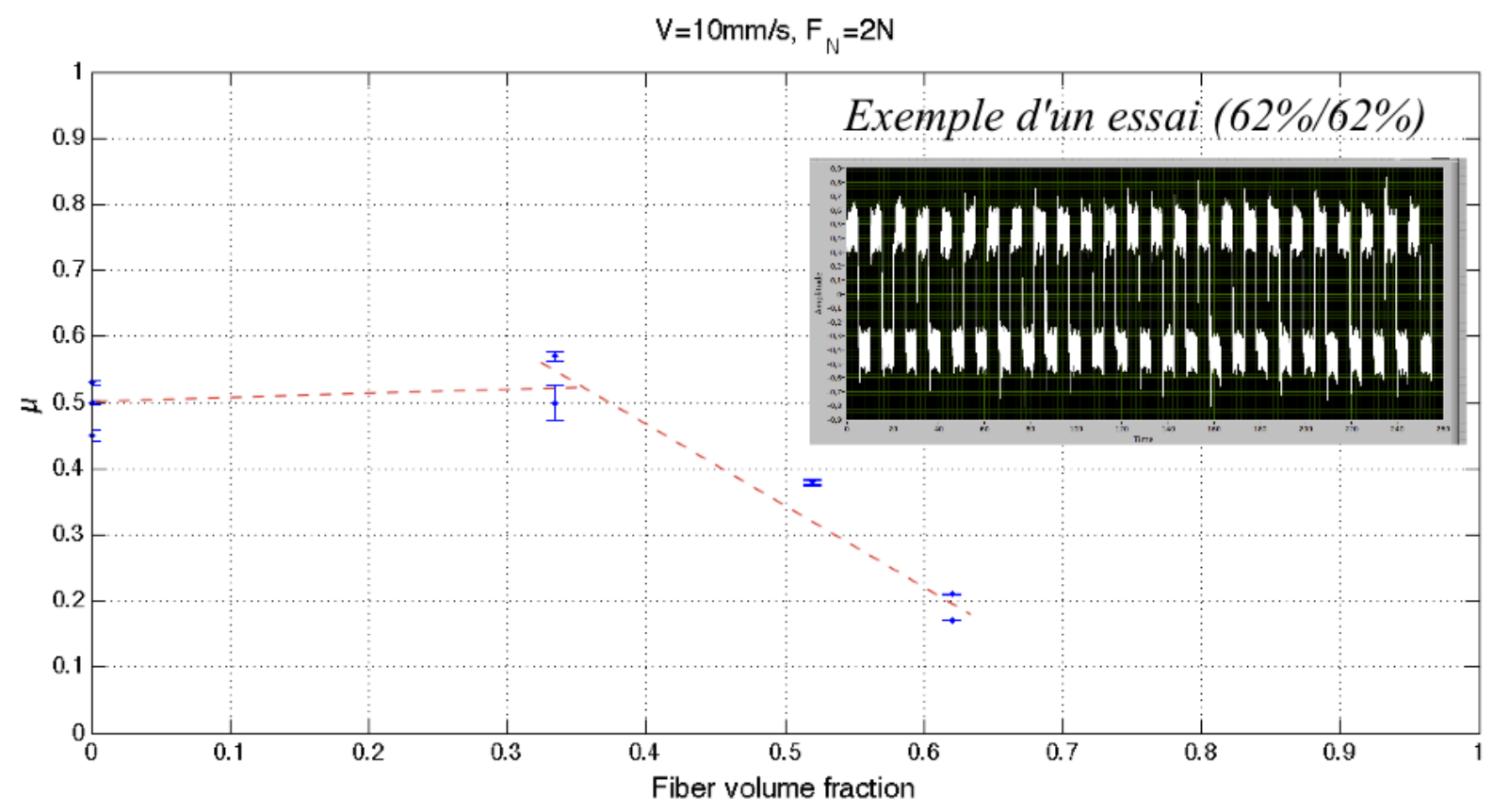

Fig. 3. Effet de la fraction volumique de fibres.

Pour des essais réalisés entre matériaux identiques, la fig. 3 montre que le frottement est stable aux faibles fractions volumiques puis diminue pour une fraction volumique supérieure à $30 \%$.

Les résultats de l'effet de l'orientation de fibres sur coefficient de frottement sont présentés à la fig. 4. Ces essais sont réalisés pour des composites de $62 \%$ sous charge normale de $18 \mathrm{~N}$ et aucune usure n'a été détectée. On caractérise l'orientation par rapport à un angle $\varphi$, qui est la somme des angles entre l'orientation de fibres de la piste ou du glisseur et le vecteur de vitesse de glissement. Par exemple, si les deux échantillons sont parallèles au sens de glissement, $\varphi=0^{\circ}$, et si ils sont tous les deux perpendiculaires au sens de glissement, $\varphi=180^{\circ}$. Entre ces deux valeurs on observe une faible augmentation du coefficient de frottement, de 0.2 à 0.25 . 


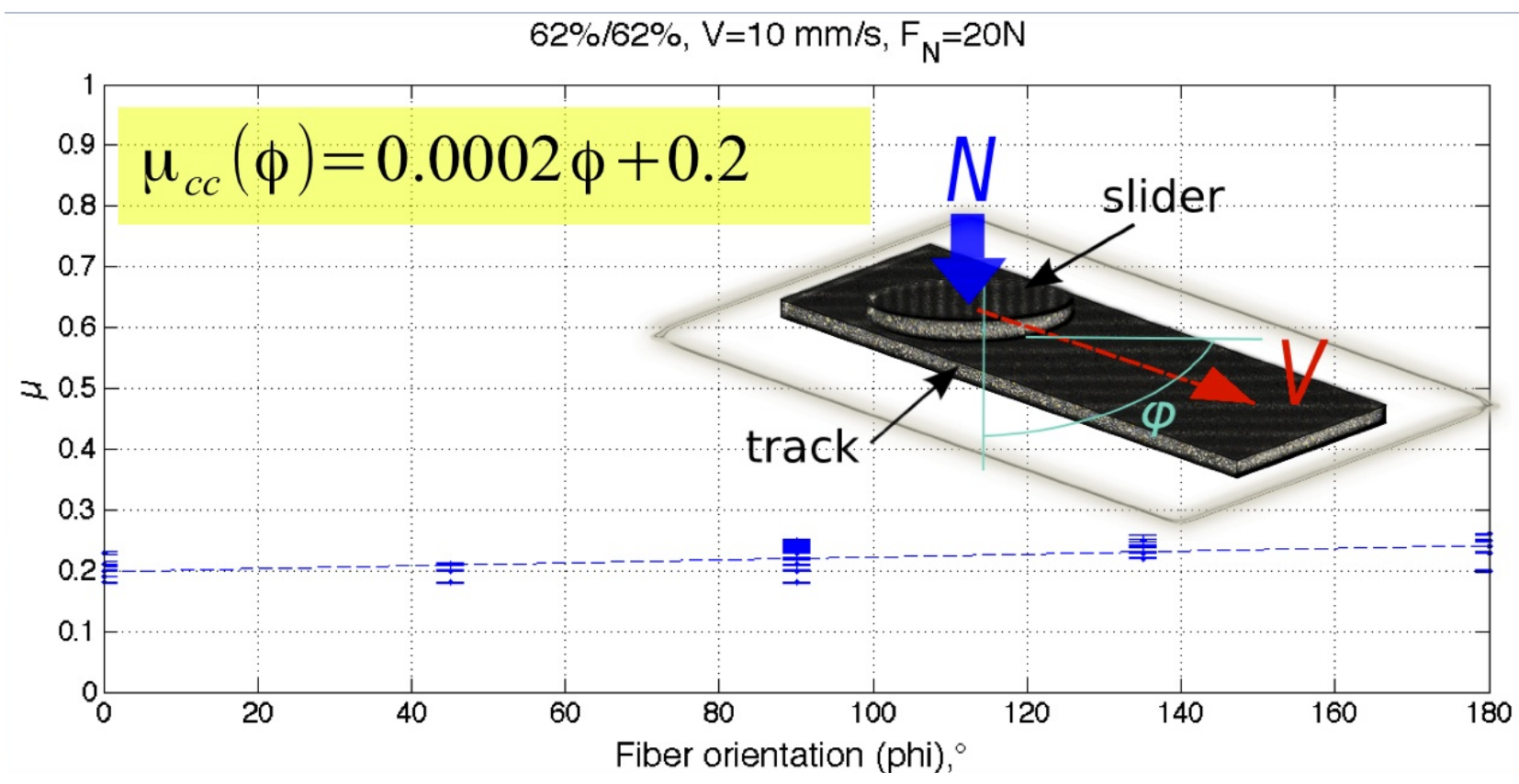

Fig. 4. Effet de l'orientation de fibres.

\section{Modèle analytique du contact de matériaux composites fibreux}

\subsection{Modèle de composite fibreux. Description de la surface}

La description classique de matériau composite à renfort de fibres longues s'appuie sur la fraction volumique de fibres, rapport entre le volume de fibres et celui de la matrice. Cependant, dans une étude de frottement interfacial, la caractéristique qui joue est la fraction surfacique de fibres. Il est donc nécessaire de s'interroger quant au rapport entre ces deux caractéristiques.

Ce dernier dépend de la distribution des fibres en volume de composite, qui peut être régulière ou aléatoire. Ce problème est connu dans le domaine de modélisation des matériaux composites afin de calculer ces caractéristiques mécaniques à partir des celles de fibres et de matrice. Il existe plusieurs types du Volume Elémentaire Représentatif (VER) [12-14] pour l'homogénéisation du composite. Les plus simples VER, carré et hexagonal, ainsi que les distributions aléatoires avec le diamètre de fibre constant et varié, sont représentés à la fig. 5.

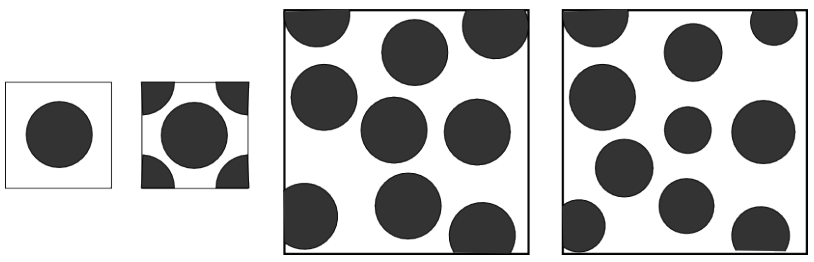

Fig. 5. VER : a) carré, b) hexagonal ; distribution aléatoire: c) $d_{f}$ constant, d) $d_{f}$ varié

Pour les caractéristiques mécaniques, modules d'élasticité et coefficients de Poisson, dans le cas de composite époxy/fibre de verre, il a été démontré [14] que les deux distributions aléatoires donnent des valeurs quasi identiques et plus proches des résultats expérimentaux que celles des VER. Quant aux VERs, les résultats d'une cellule hexagonale sont plus adéquats que ceux d'une cellule carrée. Pour caractériser notre composite, le VER carré, dont les coupes transversale et longitudinale sont présentées à la fig .6 a été choisi. 

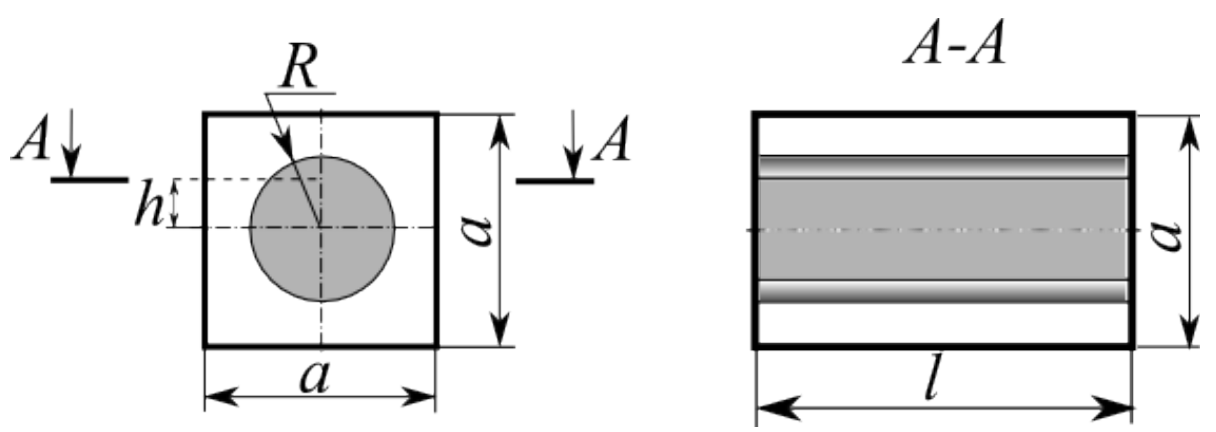

Fig. 6. Coupes transverse et longitudinale du VER carré

La fraction volumique de fibre pour le VER carré est égal à

$$
V_{f}=\frac{\pi R^{2}}{a^{2}}
$$

Pour trouver la fraction surfacique de fibre, $A_{f}$, on suppose que le plan coupant passe à la hauteur $h$ par rapport à l'axe de symétrie du VER. La densité de probabilité de cette variable aléatoire vaut 1/a. On peut trouver $A_{f}$ comme

$$
A_{f}=\frac{1}{a} \int_{-a / 2}^{a / 2} A_{f}(h) d h
$$

Suivant l'endroit où passe la coupe, $A_{f}$ est définie par rapport à $h$ comme

$$
A_{f}(h)=\left\{\begin{array}{lll}
\frac{2 \sqrt{R^{2}-h^{2}}}{a} & \text { si } & h \in[-R, R] \\
0 & \text { si } & h \notin[-R, R]
\end{array}\right.
$$

En utilisant les Eq. 4 et 5 on obtient l'expression suivante

$$
A_{f}=\frac{2}{a}\left[\int_{0}^{R} \frac{2 \sqrt{R^{2}-h^{2}}}{a} d h+\int_{R}^{a / 2} 0 d h\right]
$$

Cette expression se résout par :

$$
A_{f}=\frac{\pi R^{2}}{a^{2}}
$$

A partir des Eq. 3 et 7, dans le cas du VER carré avec coupe aléatoire, la fraction volumique de fibres est égale à la fraction surfacique de fibres.

Le modèle de matériau composite fibreux utilisé dans cette modélisation est illustré à la fig. 7. Les caractéristiques connues sont les dimensions, $b, l$ et $h$, la fraction volumique, $V_{f}$, et le diamètre d'une fibre, $d_{f}$. A partir de ce modèle on peut définir la distance moyenne entre deux fibres adjacentes :

$$
d_{m}=\frac{d_{f}}{A_{f}}\left(1-A_{f}\right)
$$




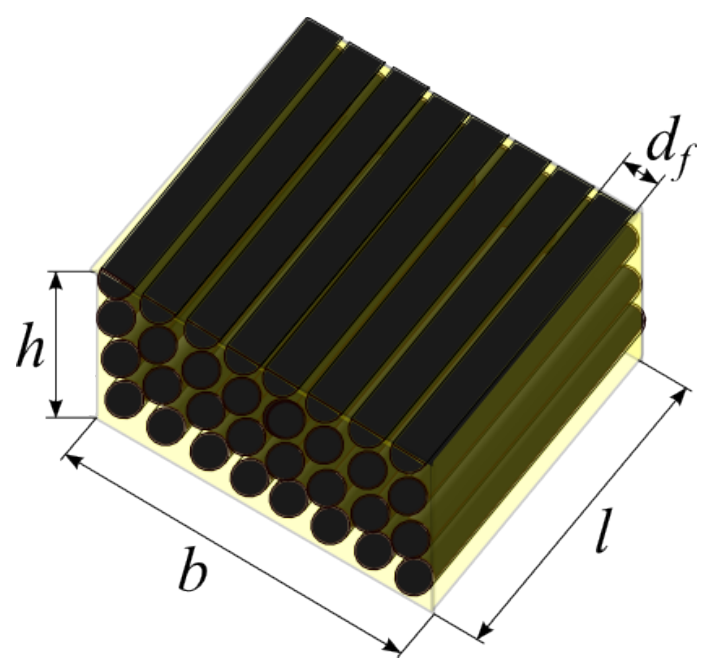

Fig. 7. Modèle du composite fibreux

Le nombre de fibres sur la surface est obtenu à partir de la géométrie de la surface :

$$
N_{f}=\frac{A_{f} b}{d_{f}}
$$

Il est nécessaire de noter que le nombre de zone de la matrice entre deux fibres sur la surface est également $N_{f}$.

\subsection{Contact et coefficient de frottement entre deux composites fibreux}

Pour décrire le frottement entre deux matériaux composites, on part de la géométrie du contact, fig.8. On présente le contact réel et le coefficient de frottement total comme une somme de microcontacts et ces coefficients de frottement sont de trois types : fibre-fibre, fibre-matrice et matrice-matrice. On suppose une contribution au frottement découplée des fibres et de la matrice. Le frottement étudié est un frottement interfacial sans usure, c'est-à-dire que les surfaces ne changent pas pendant le glissement. La dernière hypothèse du modèle est l'isotropie de frottement des fibres et de la matrice, c'est-à-dire l'indépendance du sens de glissement.

Ainsi on représente le coefficient de frottement entre deux composites comme :

$$
\mu_{\text {comp / comp }}=\mu_{f f} A_{f f}+2 \mu_{f m} A_{f m}+\mu_{m m} A_{m m}
$$

où les indices $f$ et $m$ correspondent aux fibre et à la matrice. On introduit des indices $i$ et $j$ qui désignent chacun soit $f$, soit $m$. $A_{f f}, A_{f m}$ et $A_{m m}$ sont des coefficients de contribution, calculés avec la relation suivante :

$$
A_{i j}=\frac{n_{i j} S_{i j}}{S}
$$

$S_{i j}$ est l'aire réelle moyenne d'un microcontact d'un type; $S$ est l'aire totale de tous les microcontacts. $n_{i j}$ est le nombre des microcontacts d'un type qui a priori vaut :

$$
n_{i j}=N_{i} N_{j}
$$

où $N_{i}$ et $N_{j}$ sont les nombres de fibres ou de zones de matrice en contact.

$\mathrm{Si}$ on rassemble tous ces microcontacts, on peut décrire l'aire réelle de contact, $S=b^{2} / \sin \varphi$, par le schéma présenté à la fig. 8 . 


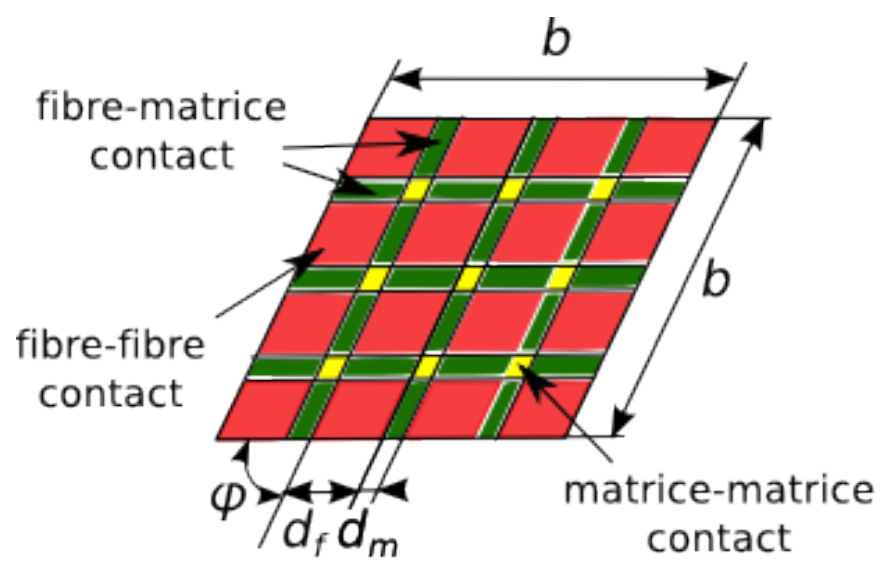

Fig. 8. Contact réel total entre deux composites

A partir de la géométrie du contact (fig. 8) et les caractéristiques de surface $d_{f}$ et $d_{m}$ (Eq. 8), l'aire réelle de chaque microcontact est définie par :

$$
S_{i j}=\frac{d_{i} d_{j}}{\sin \phi}
$$

A partir des Eq. 12 et 13 on peut ainsi identifier les coefficients de contribution :

$$
A_{i j}=\frac{n_{i j} S_{i j}}{S}=N_{i} N_{j} \frac{d_{i} d_{j}}{b^{2}}
$$

Les calculs fournissent, dans le cas de deux composites identiques, les coefficients de contribution suivants :

$$
\left\{\begin{array}{l}
A_{f f}=A_{f}^{2} \\
A_{f m}=A_{f}\left(1-A_{f}\right) \\
A_{m m}=\left(1-A_{f}\right)^{2}
\end{array}\right.
$$

Le coefficient de frottement entre deux composites est alors égal à :

$$
\mu_{\text {comp } / \text { comp }}=\mu_{f f} A_{f}^{2}+2 \mu_{f m} A_{f}\left(1-A_{f}\right)+\mu_{m m}\left(1-A_{f}\right)^{2}
$$

\subsection{Résultats du modèle}

Les hypothèses initiales du modèle reposent sur la dépendance du coefficient de frottement avec l'angle d'orientation de fibre, $\varphi$, et avec la fraction surfacique de fibres, $A_{f}$. L'Eq. 16 montre un frottement indépendant de l'angle $\varphi$. La courbe qualificative de la fraction surfacique de fibre est présentée à la fig. 9. Selon le modèle, le coefficient de frottement entre deux composites se situe sur une parabole entre des valeurs $\mu_{m m}$ et $\mu_{f f}$. 


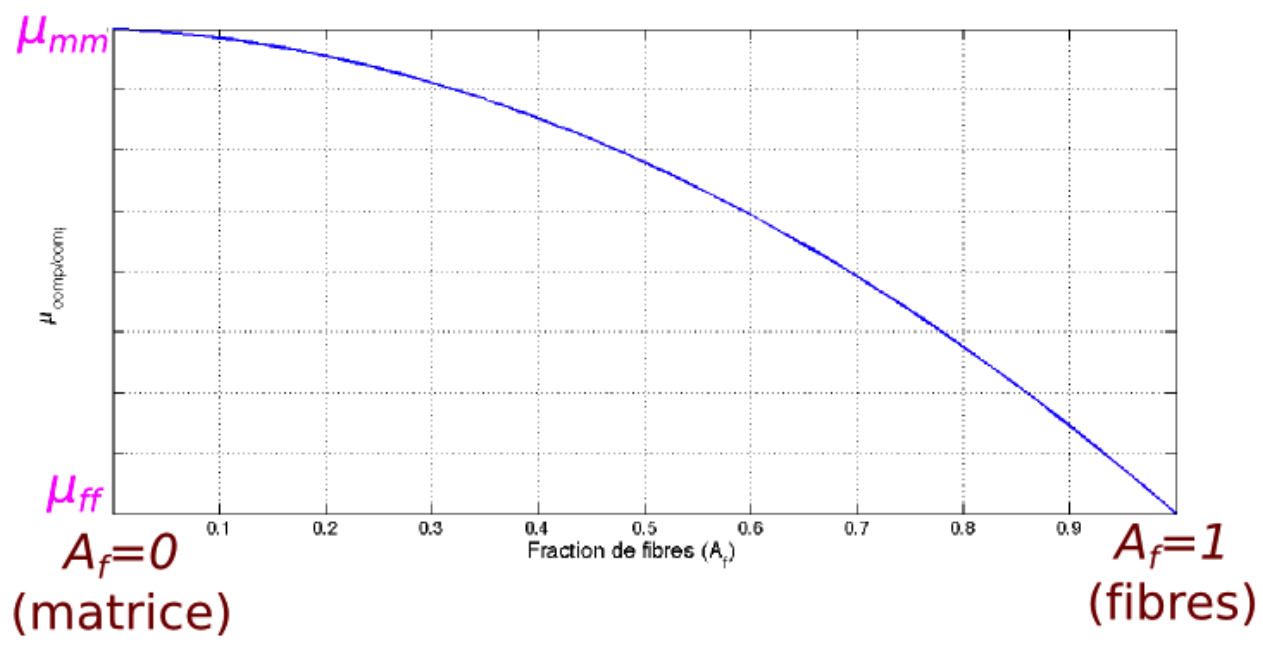

Fig. 9. Effet de la fraction surfacique de fibres

\section{Discussion et perspectives}

La dépendance de $A_{f}$ proposée par le modèle analytique correspond à la tendance déterminée pendant l'étude expérimentale. Cependant, le modèle nécessite de connaître les coefficients de frottement fibre-fibre, fibre-matrice et matrice-matrice respectivement. Alors que le coefficient pour matrice-matrice peut être obtenu expérimentalement, la réalisation d'essais pour définir les deux autres coefficients semble problématique.

L'indépendance du coefficient de frottement avec l'orientation de fibre trouvée par le modèle est relativement en accord avec les expériences : pour toute gamme de $\varphi$ de $0^{\circ}$ à $180^{\circ}, \mu$ augmente de 0.2 à 0.25 .

Compte-tenu des imperfections des matériaux utilisés dans l'étude expérimentale, une vérification de la courbe de dépendance de la fraction volumique est nécessaire. Dans ce but, les essais avec des matériaux composites à renfort de nanoparticule de carbone avec $V_{f}$ différents sont en cours de réalisation.

Dans le même temps, la question importante pour la validation du modèle est une détermination de $A_{f}$. Les méthodes d'analyse microscopique ou une modélisation analytique peuvent être appliquées. Des microphotos de coupe transverse du composite trouvées dans la littérature montrent une distribution de fibres fortement non uniforme. Cela signifie que pour la même fraction volumique, en fonction de l'endroit de passage de coupe, la variation de la fraction surfacique est très large.

\section{Conclusions}

Dans la partie expérimentale de ce travail, l'influence de l'orientation et de la fraction volumique de fibres sur la réponse au frottement entre deux composites fibreux sous faible charge normale a été montrée. Ensuite, un modèle analytique a été proposé pour estimer le coefficient de frottement. Dans le cadre de ce modèle, le problème de rapport entre fraction de fibres volumique et surfacique a été posé et discuté. Pour le cas de VER carré avec une coupe aléatoire, la fraction volumique de fibre a été trouvée égale à la fraction surfacique de fibre. La corrélation entre les résultats expérimentaux et analytiques a été analysée et jugée satisfaisante. Enfin les pistes d'amélioration du modèle et les travaux expérimentaux nécessaires à venir ont été décrits.

\section{Remerciements}

Cette étude a été financée par le Ministère des Affaires Etrangères, le Service de coopération pour la Science, la Technologie et l'Espace de l'Ambassade de France.

\section{Références}


[1] B. SURESHA G. CHANDRAMOHAN, « Effect of normal load and sliding velocity on friction and wear behavior of carbon fiber reinforced epoxy composites ». Journal of reinforced plastics and composites, Vol. 26, pp. 16951703, 2007.

[2] B.S. TRIPATHY, M.J. FUREY, « Tribological behavior of unidirectional graphile-epoxy and carbon-PEEK composites ». Wear, Vol. 162-164, pp. 385-396, 1993.

[3] T. TSUKIZOI, N. OHMAE, «Wear performance of unidirectionally oriented carbon-fiber reinforced plastics ». Tribology International, Vol. 8, pp. 171-175, 1975.

[4] H.H. SHIM, O.K. KWON, S.R. YOUN, « Friction and wear behavior of graphite fiber-reinforced composites ». Polymer Composites, Vol. 11, pp. 337-341, 1990.

[5] M. BEAUMONT, T.N. FARRIS, C.T. SUN, « Scratch testing of advanced composite surfaces ». Composites Part A, Vol. 28A, pp. 683-686, 1997.

[6] X. NING, M.R. LOVELL, « On the sliding friction characteristics of unidirectional continious FRP composites ». Journal of tribology, Vol. 124, pp. 5-13, 2002.

[7] N. SUNG, N. SUH, « Effect of fiber orientation on friction and wear of fiber-reinforced polymeric composites. ». Wear, Vol. 53, pp. 129-141, 1979.

[8] Y.N. LIANG, S.Z. LI, R. H. ZHANG. S. LI, « Effect of fiber orientation on a graphite fiber composite in single pendulum scratching ». Wear, Vol. 198, pp. 122-128, 1996.

[9] H.H. SHIM, O.K. KWON, S.R. YOUN, « Effects of fiber orientation and humidity on friction and wear properties of graphite fiber composites », Wear. Vol. 157, pp. 141-149, 1992.

[10] G. STACHOWIAK, A W BATCHELOR, « Engineering tribology ». Elsevier Butterworth-Heinemann, 2005.

[11] N. AXEN, I. M. HUTCHINGS, S. JACOBSON, « A model for the friction of multiphase materials in abrasion », Tribology International. Vol. 29, N. 6, pp. 467-475, 1996.

[12] J. RENARD, «Elaboration, microstructure et comportement des matériaux composites à matrice polymère », Lavoisier, 2005.

[13] C.T. SUN, R.S. VAIDYA, «Prediction of composite properties from a representative volume element », Composite Science and technology. Vol. 56, pp. 171-179, 1996.

[14] A. A. GUSEV, P. J. HINE, I. M. WARD, « Fiber packing and elastic properties of a transversely random unidirectional glass/epoxy composite », Composites Science and Technology. Vol. 60, pp. 535-541, 2000. 